\title{
A Modified Triples Algorithm for Flush Air Data Systems That Allows a Variety of Pressure Port Configurations
}

\author{
Daniel R. Millman, Ph.D.* \\ Booz Allen Hamilton, McLean, VA, 22102, USA
}

\begin{abstract}
Air Data Systems (FADS) are becoming more prevalent on re-entry vehicles, as evidenced by the Mars Science Laboratory and the Orion Multipurpose Crew Vehicle . A FADS consists of flush-mounted pressure transducers located at various locations on the fore-body of a flight vehicle or the heat shield of a re-entry capsule. A pressure model converts the pressure readings into useful air data quantities. Two algorithms for converting pressure readings to air data have become predominant - the iterative Least Squares State Estimator (LSSE) and the Triples Algorithm. What follows herein is a new algorithm that takes advantage of the best features of both the Triples Algorithm and the LSSE. This approach employs the potential flow model and strategic differencing of the Triples Algorithm to obtain the effective flight angles; however, the requirements on port placement are far less restrictive, allowing for configurations that are considered optimal for a FADS.
\end{abstract}

\section{Nomenclature}

$\underline{\text { Scalers }}$

$a_{i}, a_{j}, a_{k}$

$b_{i}, b_{j}, b_{k}$

$c_{i}, c_{j}, c_{k}$

$\hat{c}_{0}, \hat{c}_{1}, \hat{c}_{2}$

$c_{0}, c_{1}, c_{2}, c_{3}, c_{4}$

$a_{n, m}$

$b_{n}$

$A_{i}, A_{j}, A_{k}$

$B_{i}, B_{j}, B_{k}$

$C_{i}, C_{j}, C_{k}$

$D_{i}, D_{j}, D_{k}$

$E_{i}, E_{j}, E_{k}$

$C_{p}$

$\mathrm{M}_{\infty}$

$p_{\infty}$

$q_{c}$

$\bar{q}_{\infty}$

$u, v, w$

$V_{\infty}$

$x, y, z$ parameters from Eq. 3

parameters from Eq. 3

parameters from Eq. 3

coefficients of Eq. 17

coefficients of Eq. 22

coefficients of Eq. 28

coefficients of Eq. 27

parameters defined in Eq. 24

parameters defined in Eq. 24

parameters defined in Eq. 24

parameters defined in Eq. 24

parameters defined in Eq. 24

coefficient of pressure

freestream Mach number

freestream pressure, $p s f$

compressible dynamic pressure, $p s f$

dynamic pressure, $p s f$

velocity components, $\mathrm{ft} / \mathrm{sec}$

freestream velocity, $\mathrm{ft} / \mathrm{sec}$

position components, $f t$ angle of attack, ${ }^{\circ}$ or rads

sideslip angle, ${ }^{\circ}$ or rads

ratio of specific heats

angle of attack calibration, ${ }^{\circ}$

sideslip angle calibration, ${ }^{\circ}$

calibration factor

cone angle, rads

clock angle, rads

total flow inclination angle, rads

density, slugs

Vectors

p

$\mathbf{P}$

r

$\mathrm{V}_{\infty}$

$\mathbf{x}$

Matrices

$J$

R

II pressure readings, $p s f$

predicted pressure readings, $p s f$

radius vector, $f t$

freestream velocity vector, $\mathrm{ft} / \mathrm{sec}$

state vector

Jacobian

pressure measurement covariant matrix pressure model matrix

*Senior Aerospace Engineer, National Agencies Group - NASA, Houston, TX, Senior Member 


\section{Introduction}

$\mathrm{F}^{\text {Lush }}$ Air Data Systems (FADS) are becoming more prevalent on re-entry vehicles, as evidenced by the $\mathrm{F}$ Mars Science Laboratory ${ }^{1}$ and the Orion Multipurpose Crew Vehicle. ${ }^{2}$ A FADS consists of flush-mounted pressure transducers located at various locations on the fore-body of a flight vehicle or the heat shield of a re-entry capsule. A pressure model converts the pressure readings into useful air data quantities. Over the years, two algorithms for converting pressure readings to air data have become predominant - the iterative Least Squares State Estimator (LSSE) and the Triples Algorithm. The LSSE, developed for the Shuttle Program, ${ }^{3}$ is considered the most accurate algorithm because it uses all the pressure data simultaneously. ${ }^{4}$ The disadvantages of the LSSE is potential for convergence issues and complexity of software for real-time use. $^{5}$ In order to overcome these disadvantages, the Triples Algorithm was developed. ${ }^{6,7}$ The Triples Algorithm uses a potential flow pressure model, a cruciform pressure port configuration with the vertical ports on the line of symmetry, and a strategic set of differences to eliminate the pressures from the state vector, allowing a direct solution of the angle of attack and the sideslip angle. The potential flow model is then used to compute the freestream pressure, total pressure, and the Mach number. The Triples Algorithm does not suffer from the same convergence issues as the LSSE, but the requirement of pressure ports on the line of symmetry is often not achievable due to competing requirements, such as structural considerations, systems operations (e.g., interference with radar systems in the nose of an aircraft or missiles), or high heating rates on the line of symmetry. For example, the Orion, on Exploration Flight Test 1 (EFT-1), had to move the vertical ports off the line of symmetry due to structural issues of drilling holes on the centerline. ${ }^{2}$ This small offset eliminated the use of the Triples Algorithm for post-flight trajectory reconstruction.

What follows herein is a new algorithm that takes advantage of the best features of both the Triples Algorithm and the LSSE. This approach employs the potential flow model and strategic differencing of the Triples Algorithm to obtain the effective flight angles; however, the requirements on port placement are far less restrictive, allowing for configurations that are considered optimal for a FADS. ${ }^{8}$ Once the flight angles are known, an iterative LSSE is used to capture the requisite pressures and Mach number without resorting to a potential flow model. Finally, with the Mach number known, the true flight angles are computed. This new algorithm, which is termed the Modified Triples Algorithm (MTA), has the potential to be used as either a real-time, in-flight algorithm or for post-flight trajectory reconstruction.

In this paper, a derivation of the MTA is provided. This is followed by a discussion of pressure models that can be used to obtain freestream quantities, and the advantages and disadvantages of each approach. Comparisons are then made between NASA's Best Estimated Trajectory that includes FADS input (aeroBET) $)^{2}$ and the MTA for flight test data obtained from the Orion EFT-1. It should be noted that a US Patent Application has been filed for the MTA and its use in a FADS.

\section{Modified Triples Algorithm}

Before providing the derivation for the MTA, the coordinate axes and orientation of the flight angles must be defined. A fore-body, such as the Orion heat shield, is depicted in Fig. 1 along with the conventional body and wind axes. ${ }^{9}$ The position of the $i^{\text {th }}$ pressure port is determined from the cone angle $\lambda$ and the clock angle $\phi$, with the radius vector $\mathbf{r}_{i}$ obtained from

$$
\mathbf{r}_{i}=\left[\begin{array}{c}
x \cos \lambda_{i} \\
y \sin \phi_{i} \sin \lambda_{i} \\
z \cos \phi_{i} \sin \lambda_{i}
\end{array}\right] .
$$

The velocity vector is given by

$$
\mathbf{V}_{\infty}=\left[\begin{array}{c}
u \cos \alpha \cos \beta \\
v \sin \beta \\
w \sin \alpha \cos \beta
\end{array}\right] .
$$

The total flow inclination angle is the angle between the radius vector and the velocity vector, and is obtained by taking the dot product of the two vectors and dividing by their magnitudes, resulting in

$$
\cos \theta_{i}=\cos \alpha \cos \beta \cos \lambda_{i}+\sin \beta \sin \phi_{i} \sin \lambda_{i}+\sin \alpha \cos \beta \cos \phi_{i} \sin \lambda_{i} .
$$


From incompressible potential flow theory, for flow over a sphere, the pressure distribution is given by ${ }^{6}$

$$
C_{p}(\theta)=-\frac{5}{4}+\frac{9}{4} \cos ^{2} \theta
$$

To account for a nonspherical nose shape, compressibility, and afterbody effects (sidewash and downwash), the coefficients assume arbitrary values, while still retaining the basic form of the model,

$$
C_{p}(\theta)=A+B \cos ^{2} \theta .
$$

The values of $A$ and $B$ need to be determined for each vehicle, and the boundary condition at $\theta=0$ must be satisfied,

$$
C_{p}(0)=\frac{q_{c}}{\bar{q}_{\infty}}=A+B,
$$

Figure 1. Orion heat shield shown with body and where $q_{c}$ is the difference between the total pressure of the flow field and the free stream pressure $\left(q_{c}=\right.$
$\left.p_{0}-p_{\infty}\right)$ and is termed the compressible dynamic pressure. If the nonlinear effects are modeled into a calibration parameter $\varepsilon$, the boundary condition can be satisfied by setting

$$
A=\frac{q_{c}}{\bar{q}_{\infty}} \varepsilon,
$$

and

$$
B=\frac{q_{c}}{\bar{q}_{\infty}}(1-\varepsilon) .
$$

The calibration parameter $\varepsilon$ is determined from computational fluid dynamics (CFD), wind tunnel experiments, flight test, or a combination of the three. With these values, the pressure distribution becomes

$$
C_{p}(\theta)=\frac{q_{c}}{\bar{q}_{\infty}}\left(\cos ^{2} \theta+\varepsilon \sin ^{2} \theta\right) .
$$

Noting that the pressure coefficient for each pressure port $i$ is also given by

$$
C_{p}\left(\theta_{i}\right)=\frac{p_{i}-p_{\infty}}{\bar{q}_{\infty}},
$$

the pressure model required for the FADS becomes

$$
p_{i}=q_{c}\left(\cos ^{2} \theta_{i}+\varepsilon \sin ^{2} \theta_{i}\right)+p_{\infty} .
$$

The calibration parameter is retained in this formulation, but as will be shown in the next section, $\varepsilon$ is never evaluated. It is sufficient that $\varepsilon$ exists.

With strategic differencing, the pressure terms and calibration parameter can be removed from Eq. (11), that is ${ }^{5}$

$$
\frac{p_{i}-p_{j}}{p_{j}-p_{k}}=\frac{\cos ^{2} \theta_{i}-\cos ^{2} \theta_{j}}{\cos ^{2} \theta_{j}-\cos ^{2} \theta_{k}} .
$$

Rearranging terms, Eq. (12) is written as

$$
P_{j i} \cos ^{2} \theta_{k}+P_{i k} \cos ^{2} \theta_{j}+P_{k j} \cos ^{2} \theta_{i}=0,
$$

where

$$
\begin{aligned}
P_{j i} & =p_{j}-p_{i}, \\
P_{i k} & =p_{i}-p_{k}, \\
P_{k j} & =p_{k}-p_{j} .
\end{aligned}
$$

Equation (13) is the triples equation and will be referenced throughout this paper, although it will be written in various forms. If, for example, the three pressure port measurements being differenced are on the 
vertical meridian where the clock angle $\phi$ is either zero or $180^{\circ}$, then $\sin \phi=0$ and $\cos \phi= \pm 1$. With this simplification, all terms with $\beta$ can also be eliminated from the triples equation. The triples equation then reduces to ${ }^{5}$

$$
\tan 2 \alpha=\frac{A}{B}
$$

where

$$
\begin{aligned}
& A=P_{j i} \sin ^{2} \lambda_{k}+P_{i k} \sin ^{2} \lambda_{j}+P_{k j} \sin ^{2} \lambda_{i}, \\
& B= \pm P_{j i} \sin \lambda_{k} \cos \lambda_{k} \pm P_{i k} \sin \lambda_{j} \cos \lambda_{j} \pm P_{k j} \sin \lambda_{i} \cos \lambda_{i} .
\end{aligned}
$$

The differencing for determining $\alpha$ occurs on the vertical meridian. The ability to calculate $\alpha$ directly is due not simply to strategic differencing, but also to strict pressure port placement. Once $\alpha$ is determined, the triples equation can again be rewritten, this time to solve for $\beta$ from

$$
\hat{c}_{2} \tan ^{2} \beta+2 \hat{c}_{1} \tan \beta+\hat{c}_{0}=0,
$$

where

$$
\begin{aligned}
& \hat{c}_{2}=P_{j i} B_{k}^{2}+P_{i k} B_{j}^{2}+P_{k j} B_{i}^{2}, \\
& \hat{c}_{1}=P_{j i} A_{k} B_{k}+P_{i k} A_{j} B_{j}+P_{k j} A_{i} B_{i}, \\
& \hat{c}_{0}=P_{j i} A_{k}^{2}+P_{i k} A_{j}^{2}+P_{k j} A_{i}^{2},
\end{aligned}
$$

and

$$
\begin{array}{ll}
A_{i}=\cos \alpha \cos \lambda_{i}+\sin \alpha \cos \phi_{i} \sin \lambda_{i} & B_{i}=\sin \phi_{i} \sin \lambda_{i} \\
A_{j}=\cos \alpha \cos \lambda_{j}+\sin \alpha \cos \phi_{j} \sin \lambda_{j} & B_{j}=\sin \phi_{j} \sin \lambda_{j} \\
A_{k}=\cos \alpha \cos \lambda_{k}+\sin \alpha \cos \phi_{k} \sin \lambda_{k} & B_{k}=\sin \phi_{k} \sin \lambda_{k} .
\end{array}
$$

Now the differencing cannot occur solely on the vertical meridian. The solutions of Eq. 18 are

$$
\beta= \begin{cases}\tan ^{-1}\left(-\frac{1}{2} \frac{\hat{c}_{0}}{\hat{c}_{1}}\right) & \hat{c}_{2}=0, \\ \tan ^{-1}\left[-\left(\frac{\hat{c}_{1}}{\hat{c}_{2}}\right) \pm \sqrt{\left(\frac{\hat{c}_{1}}{\hat{c}_{2}}\right)^{2}-\left(\frac{\hat{c}_{1}}{\hat{c}_{2}}\right)}\right] & \hat{c}_{2} \neq 0,\end{cases}
$$

where the root chosen in the second case is the one closest to zero. ${ }^{5}$

While a powerful real-time algorithm, the Triples Algorithm relies on placing pressure ports exactly on the vertical meridian. An iterative approach is required in order to use the triples equation when the limitation of pressure port placement is removed. However, differencing must still occur primarily parallel to the $x-z$ plane (termed notional vertical) for determining $\alpha$, and primarily parallel to the $x-y$ plane (termed notional horizontal) for determining $\beta$. To begin the iterations, a guess is made for $\beta$. Since the sideslip angle is typically small, a reliable initial guess to start this algorithm is $\beta=0$. For a trajectory, the previously converged value of $\beta$ is used to start the iterations. The MTA equations in the following paragraphs have been submitted as part of a US Patent Application for an air data system by Booz Allen Hamilton.

For a given value of $\beta$, define the following parameters

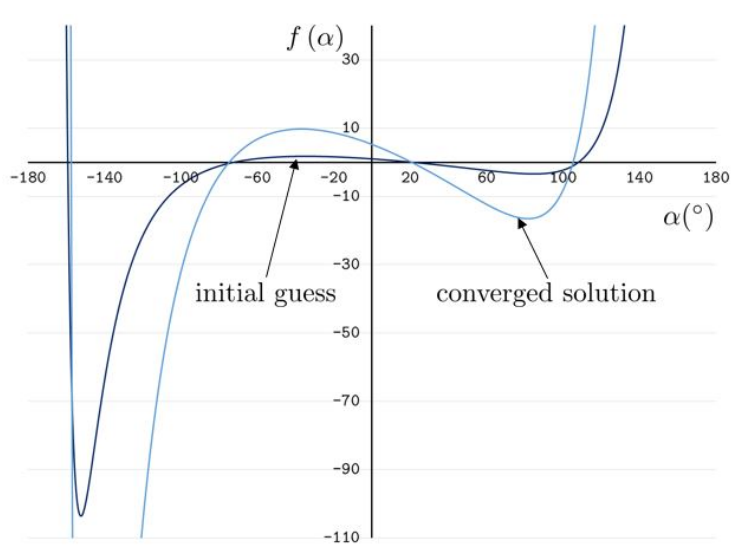

Figure 2. Typical behavior of the polynomial given in Eq. (22). from Eq. (3),

$$
\begin{array}{lll}
a_{i}=\cos \beta \cos \lambda_{i} & a_{j}=\cos \beta \cos \lambda_{j} & a_{k}=\cos \beta \cos \lambda_{k}, \\
b_{i}=\sin \beta \sin \phi_{i} \sin \lambda_{i} & b_{j}=\sin \beta \sin \phi_{j} \sin \lambda_{j} & b_{k}=\sin \beta \sin \phi_{k} \sin \lambda_{k}, \\
c_{i}=\cos \beta \cos \phi_{i} \sin \lambda_{i} & c_{j}=\cos \beta \cos \phi_{j} \sin \lambda_{j} & c_{k}=\cos \beta \cos \phi_{k} \sin \lambda_{k} .
\end{array}
$$


After some modest algebra and judicial use of trigonometric identities, it can be shown (see the Appendix) that the triples equation becomes

$$
c_{4} \tan ^{4} \frac{1}{2} \alpha+c_{3} \tan ^{3} \frac{1}{2} \alpha+c_{2} \tan ^{2} \frac{1}{2} \alpha+c_{1} \tan \frac{1}{2} \alpha+c_{0}=0,
$$

where

$$
\begin{aligned}
& c_{4}=P_{j i} A_{k}+P_{i k} A_{j}+P_{k j} A_{i}, \\
& c_{3}=P_{j i} B_{k}+P_{i k} B_{j}+P_{k j} B_{i}, \\
& c_{2}=P_{j i} C_{k}+P_{i k} C_{j}+P_{k j} C_{i}, \\
& c_{1}=P_{j i} D_{k}+P_{i k} D_{j}+P_{k j} D_{i}, \\
& c_{0}=P_{j i} E_{k}+P_{i k} E_{j}+P_{k j} E_{i},
\end{aligned}
$$

and

$$
\begin{array}{lll}
A_{i}=\left(a_{i}-b_{i}\right)^{2} & A_{j}=\left(a_{j}-b_{j}\right)^{2} & A_{k}=\left(a_{k}-b_{k}\right)^{2}, \\
B_{i}=4 c_{i}\left(b_{i}-a_{i}\right) & B_{j}=4 c_{j}\left(b_{j}-a_{j}\right) & B_{k}=4 c_{k}\left(b_{k}-a_{k}\right), \\
C_{i}=2\left(b_{i}^{2}+2 c_{i}^{2}-a_{i}^{2}\right) & C_{j}=2\left(b_{j}^{2}+2 c_{j}^{2}-a_{j}^{2}\right) & C_{k}=2\left(b_{k}^{2}+2 c_{k}^{2}-a_{k}^{2}\right), \\
D_{i}=4 c_{i}\left(b_{i}+a_{i}\right) & D_{j}=4 c_{j}\left(b_{j}+a_{j}\right) & D_{k}=4 c_{k}\left(b_{k}+a_{k}\right), \\
E_{i}=\left(a_{i}+b_{i}\right)^{2} & E_{j}=\left(a_{j}+b_{j}\right)^{2} & E_{k}=\left(a_{k}+b_{k}\right)^{2} .
\end{array}
$$

Equation (22) has been found to have the desirable properties of well spaced roots, approximately $90^{\circ}$ apart, and well behaved near the root of interest (see Fig. 2). Also, the singularities have been moved to $\pm 180^{\circ}$. Quadratic convergence can also be achieved for evaluating the root of interest using the NewtonRaphson method. An overview of the MTA is depicted in Fig. 3.

\section{Averaging Method}

The MTA, like the Triples Algorithm, averages the results of every flight angle obtained from each differencing. For example, if there are five ports in the notional vertical, then there are

$$
\frac{5 !}{3 !(5-3) !}=10
$$

angles of attack to average. While a $\chi^{2}$ approach has been used for health monitoring, ${ }^{10,11}$ a different approach was used here. As each value of $\alpha$ is computed, a check is made for convergence to a real number. If a $\mathrm{NaN}$ (not a number) is detected or convergence is not achieved in a set number of iterations, iterations are stopped and that value is scored as invalid. After all $\alpha$ 's are computed, only the valid angles of attack are averaged. Additionally, the standard deviation is computed. A second averaging of the valid $\alpha$ 's is made, but outliers (values outside $\pm 3 \sigma$ ) are removed from the averaging. The same technique is used when averaging $\beta$.

\section{Angle Calibrations}

As with the Triples Algorithm, Eqs. (20) and (22) provide effective flight angles, $\alpha_{e}$ and $\beta_{e}$. These angles will differ from the true flight angles, $\alpha_{t}$ and $\beta_{t}$, since the potential flow model from which the equations were derived is only approximate. A calibration procedure is necessary in order to retrieve the true flight angles from Eqs. (20) and (22). ${ }^{6}$ A database of the coefficients of pressure at each of the port locations and selected Mach numbers, angles of attack, and sideslip angles is required. Using the above equations, the effective angles are computed, and the calibration corrections are obtained from

$$
\begin{aligned}
\delta \alpha\left(M_{\infty}, \alpha_{e}\right) & =\alpha_{e}-\alpha_{t}, \\
\delta \beta\left(M_{\infty}, \alpha_{e}, \beta_{e}\right) & =\beta_{e}-\beta_{t} .
\end{aligned}
$$


Typically, for the Orion database, a fourth order polynomial is required to approximate the relationship between the calibration angle $\delta \alpha$ and the effective angle of attack $\alpha_{e}$ at each Mach number. Once a Mach number is determined, the procedure of which will be described in the next section, an interpolation is performed on the coefficients of the fourth order polynomial in order to determine the $\delta \alpha$. This calibration angle is subtracted from the effective angle of attack in order to deter-

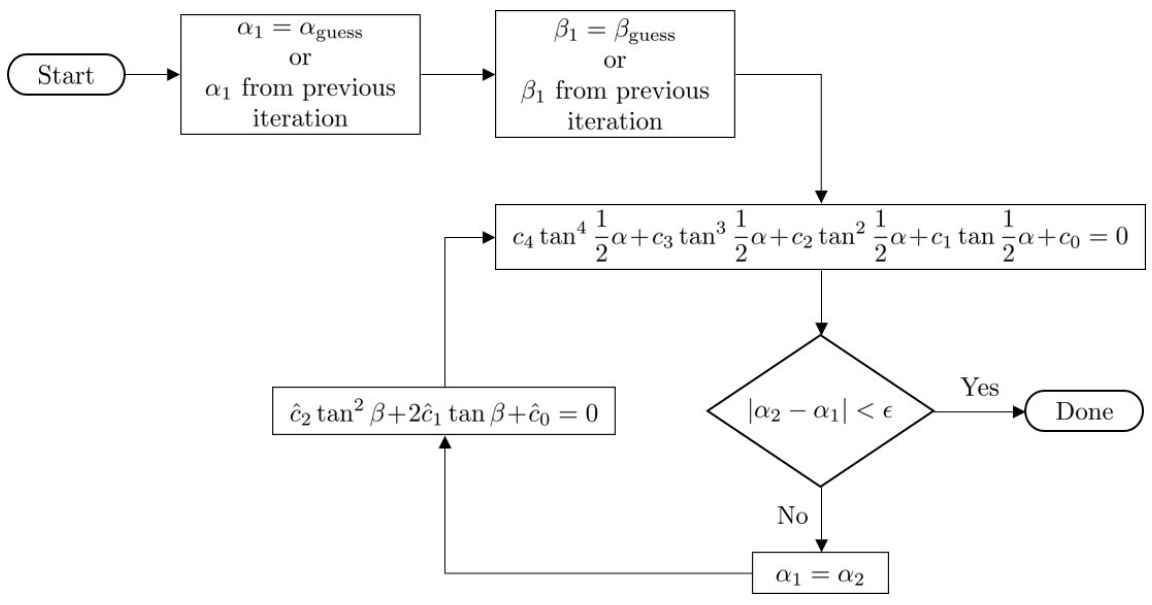

Figure 3. Modified Triples Algorithm. mine the true angle of attack $\alpha_{t}$.

Determining the true sideslip angle $\beta_{t}$ is more problematic. At each Mach number, very different curves exist between each calibration angle $\delta \beta$ and the effective sideslip angle $\beta_{e}$ at each true angle of attack $\alpha_{t}$. Additionally, these curves are nearly linear at subsonic and hypersonic Mach numbers, but are cubic at transonic to high supersonic Mach numbers. In order to simplify computations, the relationship between the coefficients of the curves between the sideslip calibration angles and the effective sideslip angles at each Mach number was determined as a polynomial with the true angle of attack. For any Mach number in the database, the correlation takes the form

$$
\delta \beta=\sum_{n=0}^{N} b_{n} \beta_{e}^{n},
$$

where

$$
\begin{gathered}
b_{0}=\sum_{m=0}^{M} a_{0, m} \alpha_{t}^{m} \\
\vdots \\
b_{N}=\sum_{m=0}^{M} a_{N, m} \alpha_{t}^{m} .
\end{gathered}
$$

The stored coefficients $a_{n, m}$ are now functions of just Mach number and $\alpha_{t}$. The calibration angle $\delta \beta$ is determined at the Mach numbers in the database above and below the given Mach number though the following procedure. The coefficients $a_{n, m}$ are determined at each Mach number, and with the coefficients $a_{n, m}$, the coefficients $b_{n}$ are determined. From the coefficients $b_{n}$ and the effective sideslip angles the calibration angles at each Mach number are determined. The calibration angle $\delta \beta$ is determined by interpolating between the two Mach numbers. The calibration angle is subtracted from the effective sideslip angle to obtain the true sideslip angle $\beta_{t}$.

\section{Pressure Calculations}

The calibrations cannot be applied until the Mach number is obtained. The procedures to do so is now described. Typically, in a Pitot-static system, the two pressures that are directly measured are the total pressure, $p_{0}$, and the freestream pressure, $p_{\infty}$. However, to close the FADS equations, any two pressures with a Mach number relationship are sufficient. As with MEADS ${ }^{12}$ and the Orion EFT-1 FADS, ${ }^{2}$ dynamic pressure was chosen over the compressible dynamic pressure. Since dynamic pressure can be expressed as 
either

$$
\begin{aligned}
\bar{q} & =\frac{1}{2} \rho_{\infty} V_{\infty}^{2}, \text { or } \\
\bar{q} & =\frac{1}{2} \gamma p_{\infty} \mathrm{M}_{\infty}^{2} .
\end{aligned}
$$

Both equations are extremely useful for freestream pressure and Mach number determination, and are far easier to implement than the complicated relationship between the total pressure and freestream pressure.

Two approaches are available for calculating the freestream quantities. In the first approach, only FADS pressure data are used to estimate the freestream and dynamic pressure. This approach is useful for Mach numbers less than Mach 2. For high Mach numbers, where $\bar{q}_{\infty} \gg p_{\infty}$, another approach is required . For the second approach, FADS pressure data are used to obtain $\bar{q}_{\infty}$. Either the inertial velocity, balloon data, and an atmosphere model are used to estimate $p_{\infty}$, or the freestream pressure can be estimated from the GPS altitude..

\section{A. Pressure Model 1 (PM1) - FADS Only Approach}

The atmospheric state vector is defined as $\mathbf{x}=\left[\bar{q}_{\infty}, p_{\infty}\right]^{T}$. For each pressure port, the pressure model takes the form

$$
P_{i}=\bar{q}_{\infty} C_{p_{i}}\left(\mathrm{M}_{\infty}, \alpha_{e}, \beta_{e}\right)+p_{\infty},
$$

where the pressure coefficient $C_{p}$ is a function only of the freestream Mach number and the effective flight angles. This is a different pressure model than was used to derive the MTA. One of the benefits of employing this pressure model is the calibration parameter, $\varepsilon$, need not be computed. Much of the following discussion follows Refs. [1] and [2], although the state vector is greatly simplified. Additionally, the database of the coefficient of pressures, which is obtained from CFD or wind tunnels with true flight angles, must be remapped to the effective flight angles.

The pressure model can be written in vector notation as

$$
\mathbf{P}=\Pi \mathbf{x}
$$

where

$$
\boldsymbol{\Pi}=\left[\begin{array}{cc}
C_{p}\left(\mathrm{M}_{\infty}, \theta_{1}\right) & 1 \\
\cdot & \cdot \\
\cdot & \cdot \\
\cdot & \cdot \\
C_{p}\left(\mathrm{M}_{\infty}, \theta_{N}\right) & 1
\end{array}\right] .
$$

If the vector of $N$ pressure readings at time $t$ is defined as $\mathbf{p}$, the pressure measurement model can be rewritten as

$$
\mathbf{p}=\mathbf{P}+\boldsymbol{\epsilon},
$$

where $\epsilon$ represents the pressure measurement errors. A linearization of the pressure model is represented by

$$
\mathbf{p} \approx \mathbf{P}(\overline{\mathbf{x}})+\left.\frac{\partial \mathbf{P}}{\partial \mathbf{x}}\right|_{\mathbf{x}=\overline{\mathbf{x}}}(\mathbf{x}-\overline{\mathbf{x}})+\boldsymbol{\epsilon}
$$

where $\overline{\mathbf{x}}$ is a reference state. Let $\boldsymbol{J}$ represent the Jacobian,

$$
\boldsymbol{J}(\overline{\mathbf{x}})=\left.\frac{\partial \mathbf{P}}{\partial \mathbf{x}}\right|_{\mathbf{x}=\overline{\mathbf{x}}} .
$$

Then

$$
\mathbf{p}-\mathbf{P}+\boldsymbol{J}(\overline{\mathbf{x}}) \overline{\mathbf{x}}=\boldsymbol{J}(\overline{\mathbf{x}}) \mathbf{x}+\boldsymbol{\epsilon}
$$

By defining

$$
\mathbf{y} \equiv \mathbf{p}-\mathbf{P}+\boldsymbol{J}(\overline{\mathbf{x}}) \overline{\mathbf{x}},
$$

then Eq. (38) becomes

$$
\mathbf{y}=\boldsymbol{J}(\overline{\mathbf{x}}) \mathbf{x}+\boldsymbol{\epsilon} .
$$


Per the Gauss-Markov theorem, the best linear unbiased estimate of $\mathbf{x}$ is the weighted least-squares solution

$$
\hat{\mathbf{x}}=\left(\boldsymbol{J}^{T} \boldsymbol{R}^{-1} \boldsymbol{J}\right)^{-1} \boldsymbol{J}^{T} \boldsymbol{R}^{-1} \mathbf{y},
$$

where $\boldsymbol{R}$ is the pressure measurement covariance matrix. Keeping in mind that the database of $C_{p}$ is a function only of Mach number, $\alpha_{e}$, and $\beta_{e}$, the Jacobians are given by

$$
\begin{aligned}
J_{i, 1} & =\frac{\partial p_{i}}{\partial \bar{q}_{\infty}}=C_{p_{i}}\left(\mathrm{M}_{\infty}, \alpha_{e}, \beta_{e}\right), \\
J_{i, 2} & =\frac{\partial p_{i}}{\partial p_{\infty}}=1 .
\end{aligned}
$$

With these definitions of the Jacobians, the vector $\mathbf{y}$ reduces to the measured pressures $\mathbf{p}$. Additionaly, if the errors in the pressure ports are considered independent of each other, the covariance matrix reduces to the identity matrix multiplied by an error estimate. The matrix $\left(\boldsymbol{J}^{T} \boldsymbol{R} \boldsymbol{J}\right)$ is easily inverted, the error estimate is eliminated, and the solutions for the freestream values are simply

$$
\begin{aligned}
\bar{q}_{\infty} & =\frac{N\left(\sum_{i=1}^{N} C_{p_{i}} p_{i}\right)-\left(\sum_{i=1}^{N} C_{p_{i}}\right)\left(\sum_{i=1}^{N} p_{i}\right)}{N\left(\sum_{i=1}^{N} C_{p_{i}}^{2}\right)-\left(\sum_{i=1}^{N} C_{p_{i}}\right)^{2}}, \\
p_{\infty} & =\frac{\left(\sum_{i=1}^{N} C_{p_{i}}^{2}\right)\left(\sum_{i=1}^{N} p_{i}\right)-\left(\sum_{i=1}^{N} C_{p_{i}}\right)\left(\sum_{i=1}^{N} C_{p_{i}} p_{i}\right)}{N\left(\sum_{i=1}^{N} C_{p_{i}}^{2}\right)-\left(\sum_{i=1}^{N} C_{p_{i}}\right)^{2}} .
\end{aligned}
$$

All that is required to get the iterations started is a guess of the Mach number. The Mach number is updated from

$$
\mathrm{M}_{\infty}=\sqrt{\frac{2 \bar{q}_{\infty}}{\gamma p_{\infty}}}
$$

\section{B. Pressure Model 2 (PM2) - FADS with INS/GPS Aiding}

For the second approach, it is noted, as shown in the Results section, that the MTA has the ability to capture flight angles at high altitudes and high Mach numbers. The differencing employed by the MTA also provides the simplest of filters to remove noise. If it assumed that the noise from each pressure port is of the same order of magnitude, than differencing should also provide more accurate predictions for pressure. For example, for the $\mathrm{i}^{\text {th }}$ and $\mathrm{j}^{\text {th }}$ pressure ports,

$$
\begin{aligned}
p_{i} & =\bar{q}_{\infty} C_{p_{i}}\left(\mathrm{M}_{\infty}, \alpha_{e}, \beta_{e}\right)+p_{\infty}, \\
p_{j} & =\bar{q}_{\infty} C_{p_{j}}\left(\mathrm{M}_{\infty}, \alpha_{e}, \beta_{e}\right)+p_{\infty} .
\end{aligned}
$$

Letting

$$
\begin{aligned}
\Delta p_{i j} & =p_{i}-p_{j}, \\
\Delta C_{p_{i j}} & =C_{p_{i}}-C_{p_{j}},
\end{aligned}
$$

and differencing Eqs. (45a) and (45b) results in

$$
\Delta p_{i j}=\bar{q}_{\infty} \Delta C_{p_{i j}}
$$

with the least squares solution of

$$
\bar{q}_{\infty}=\frac{\sum_{i=1}^{N-1} \sum_{j=i+1}^{N} \Delta C_{p_{i j}} \Delta p_{i j}}{\sum_{i=1}^{N-1} \sum_{j=i+1}^{N}\left(\Delta C_{p_{i j}}\right)^{2}} .
$$

Unfortunately, the freestream pressure is eliminated in the differencing. However, using inertial velocity, GPS altitude, a temperature profile, and winds aloft, an estimate of the freestream pressure is computed, 


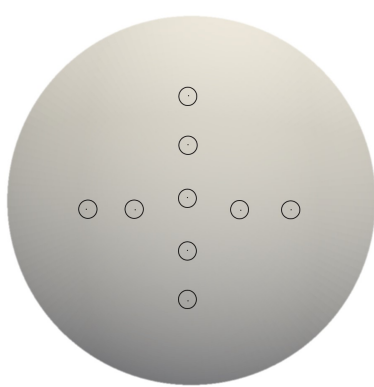

(a) Cruciform

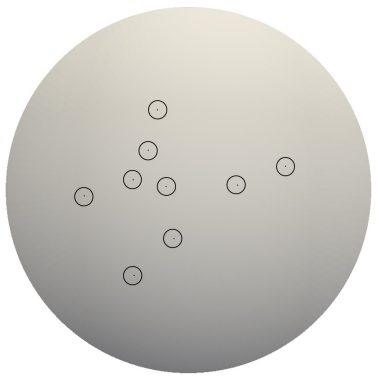

(d) Staggered

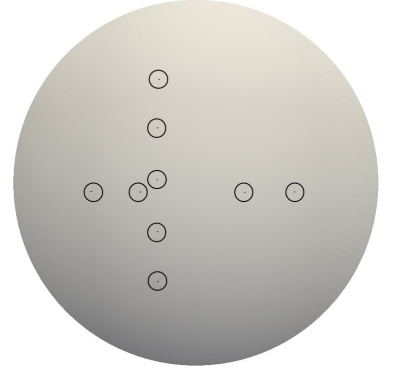

(b) Slightly Offset

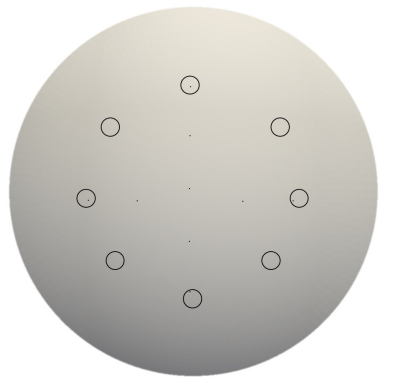

(e) Annular

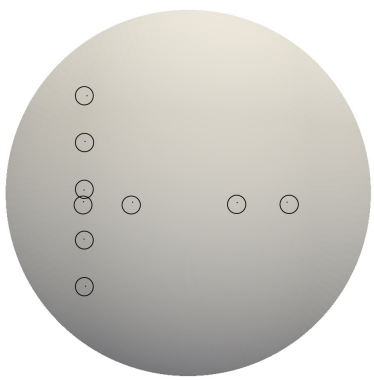

(c) Offset

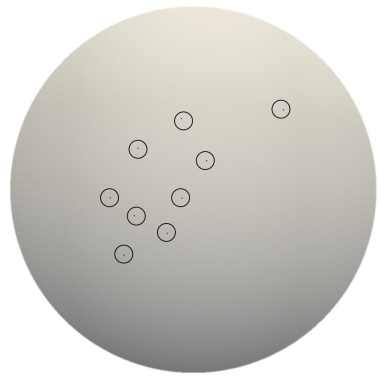

(f) Random

Figure 4. A variety of configurations for which MTA has been successfully applied.

and the Mach number is iterated until convergence. ${ }^{2,12}$ As in the first approach, a guess is made for the Mach number, but now the dynamic pressure is solved from Eq. (48). From the inertial velocity, GPS altitude, and winds aloft, provided by an atmosphere model or balloon data, an estimate of the true airspeed, $V_{\infty}$, is obtained, and

$$
\rho_{\infty}=\frac{2 \bar{q}_{\infty}}{V_{\infty}^{2}}
$$

From the GPS altitude and temperature profile, again provided by an atmosphere model or balloon data, the freestream pressure is obtained from

$$
p_{\infty}=\rho_{\infty} \mathrm{R} T_{\infty} .
$$

A new estimate for the Mach number is obtained from Eq. (44), and the iterations continue until reaching a specified level of convergence.

\section{Pressure Model 3 (PM3) - FADS with GPS Aiding}

The above method can be used in a real-time approach if the latest balloon data or atmosphere model is loaded into the re-entry vehicle prior to re-entry. If balloon data are not available, reasonable results can still be obtained by converting the GPS altitude directly into a freestream pressure estimate. Both approaches are examined in the results section.

\section{Results}

In order to test the efficacy of the algorithm, a model problem was formulated. A geometry roughly equivalent to the the Orion heat shield was generated. Given a pressure altitude, Mach number, angle of attack, and sideslip angle, Eq. (11) was used to generate a pressure distribution on the heat shield. Since perfect data were provided to the MTA, the MTA should reproduce the flight angles perfectly given a reasonable guess of the angle of attack and sideslip angle, regardless of the port configuration. This was indeed found to be the case. Inner and outer loop iterations never exceeded seven, even with a tolerance of $10^{-10}$. The various port configurations that the MTA successfully reproduced the flight angles are shown in Fig. 4. The annular configuration of Fig. $4(\mathrm{e})$ is of particular interest as studies indicate this configuration may be optimal for FADS. ${ }^{8}$ The initial guess on the flight angles were typically $\alpha=20^{\circ}$ and $\beta=0$, although 
even with a guess of $\alpha=0$ and $\beta= \pm 30^{\circ}$, the MTA converged rapidly, indicating the utility of the MTA for various configurations.

To determine the robustness of the MTA and the different pressure models in the presence of noise, i.e. imperfect data, the algorithm was used to reconstruct the trajectory of the Orion EFT-1. Goodness of the algorithm was determined by how well it agreed with NASA's aeroBET. Some differences between how the MTA was applied and how NASA obtained its aeroBET should be noted. The aeroBET was derived from radar, inertial, and FADS data, all combined through an extended Kalman filter. FADS pressure data were smoothed where significant dropouts and sensor saturation occurred prior to the LSSE algorithm being applied. Additionally, after results were obtained, a Butterworth low pass filter was applied to further smooth the results. ${ }^{2}$ In contrast, no attempt to smooth the pressure data was made when applying the MTA. Additionally, no post-computation smoothing was applied to the results. So the MTA results are noisier than the aeroBET, and more indicative of an unfiltered, realtime solution. For more information on the NASA predictions, including the pressure data information

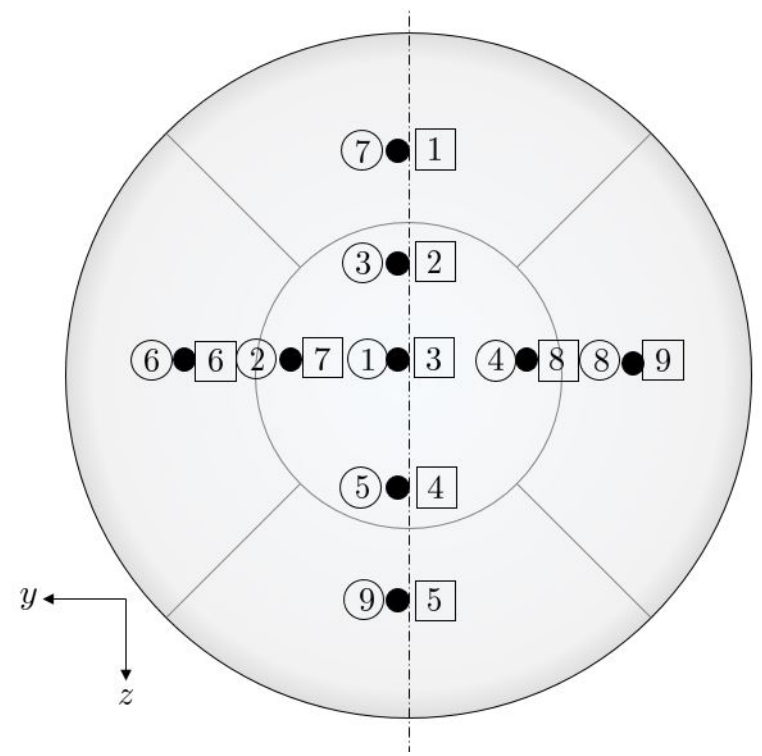

Figure 5. Pressure port numbering on the Orion heat shield. obtained from the FADS, refer to Ref. [2].

A representation of the Orion heat shield is depicted in Fig. 5. The numbers to the left of the pressure ports (in circles) is the numbering system used in Ref. [2]. The numbers to the right of the pressure ports is the numbering system used in the present study. For the LSSE, the numbering does not matter as all pressure data is used simultaneously. For the Triples Algorithm, NASA's numbering system is useful since all the vertical ports are odd numbers and all the horizontal ports are even numbers. This numbering system is very effective when setting up the differencing required in the Triples Algorithm. However, since the vertical ports are offset from the vertical meridian, the Triples Algorithm as written could not be used. The numbers to the right of the pressure ports provide an effective system for differencing with the MTA. Not only are the ports in vertical used to compute $\alpha$, but ports off the vertical can also be used, providing more differences than is allowed in the Triples algorithm. In fact, if the ports were on the vertical meridian, a total of 10 differences can be used in the Triples Algorithm. With the MTA, there are 50 differences that are available to estimate $\alpha$ and 34 differences available to estimate $\beta$. The fact that so many differences are now available adds more reliability to the FADS since the loss of one or two ports does not significantly degrade the ability to compute the flight angles. Also, since the cruciform configuration is no longer required, more configurations can be examined that are optimal for the vehicle being design.

Before examining the full trajectory reconstruction, it is useful to look at the flight angle predictions without calibrations applied. Figure 6 shows the how the aeroBET and the MTA predictions of $\alpha$ and $\beta$ trended throughout the trajectory, and the percent difference/absolute difference between the two predictions. Without the calibrations applied, it is not expected that the MTA will provide the same predictions as aeroBET. The MTA begins to stabilize towards valid predictions at about 60 seconds of elapsed time from entry interface, but a large spike is observed from about 100 to 110 seconds. Two of the pressure sensors in this region are saturated at full values, ${ }^{2}$ leading to the large error in the flight angle predictions. After the sensors are no longer saturated at full value, the predictions again begin to stabilize. Examination of the calibration data indicated that with a good Mach number prediction, the MTA predictions should compare well with the aeroBET. At about 110 seconds elapsed time, Orion EFT-1 is still well above 200,000 ft and faster than Mach 25.

The predicted effective flight angles shown in Fig. 6 are used in all of the pressure models. Results from PM1 are shown in Fig. 7. Predictions were invalid until the flow was at low supersonic numbers, and results are shown from 280 seconds elapsed time. Other authors have noted the issues with numerical stability using just pressure data at supersonic speeds, ${ }^{2,12}$ but what is interesting is the large deltas at supersonic 


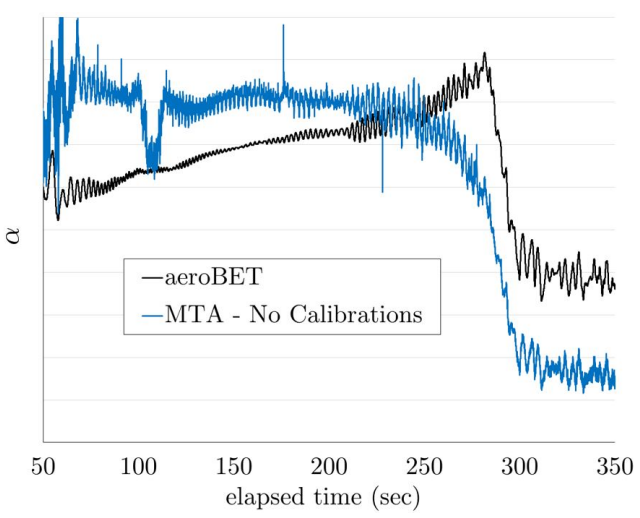

(a) Trending information

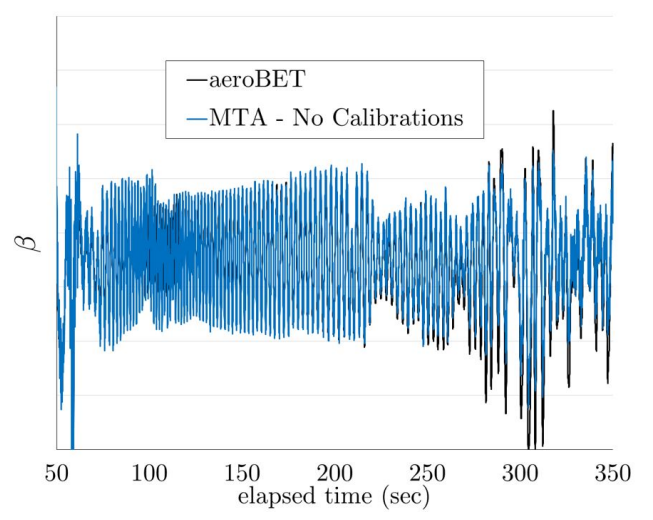

(c) Trending information

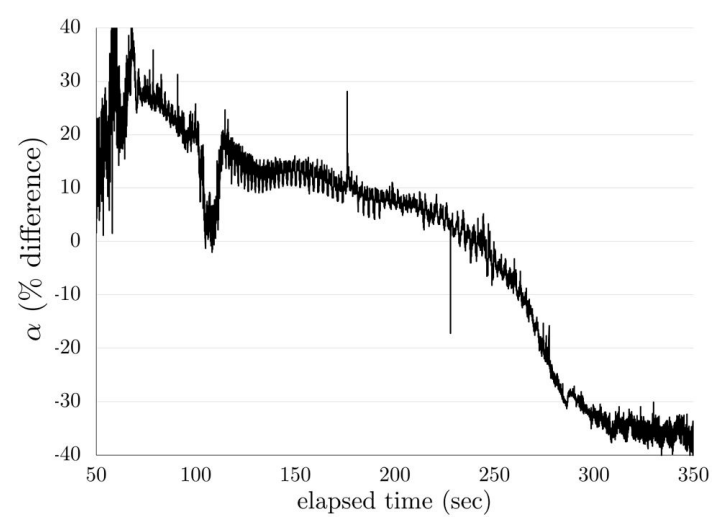

(b) Percent difference

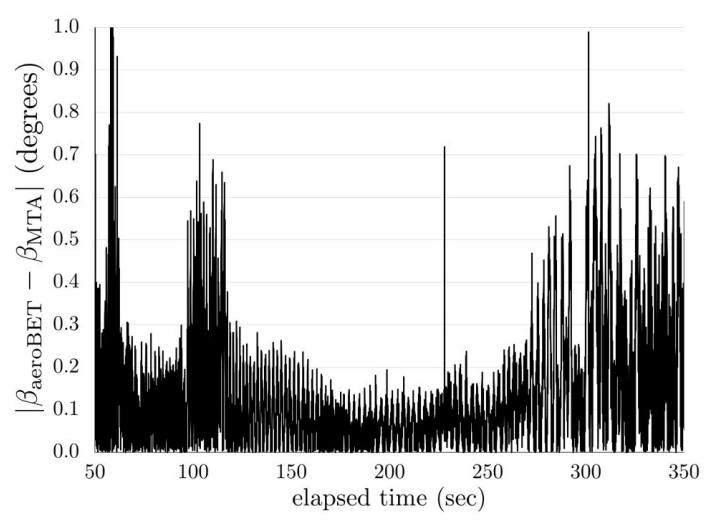

(d) Absolute difference

Figure 6. Comparison of $\alpha$ and $\beta$ between the aeroBET and the MTA with no calibrations applied.

speeds and the nearly perfect correlations at subsonic speeds. A plausible explanation would be that the supersonic database is in poor agreement with actual flight conditions due to geometry changes caused by ablation during re-entry. Unpredicted shock wave structure could lead to an predicted pressure distribution. Once the shock waves subside, the database predictions and actual pressure distribution are more closely in agreement.

In order to overcome the lack of data during the high speed portion of the trajectory, the FADS requires aiding from other systems. Results from PM2 are shown in Fig. 8. Again, correlations between the aeroBET and MTA with PM2 are in excellent agreement during the latter part of the trajectory where conditions are subsonic. The Mach number is in excellent agreement throughout the entire trajectory. This should not be surprising. Since PM2 assumes both the freestream velocity and temperature from the inertial and balloon data are correct, this is equivalent to saying the Mach number is known. The dynamic pressure data, which comes solely from the FADS, is in very good agreement throughout the hypersonic/supersonic portion of the trajectory. Since the deltas in the Mach number are small, the errors in the dynamic pressure and freestream pressures are the same, due to the simple relationship between the two. Again, the unpredicted shock structure due to ablation could be leading to the different results between the aeroBET and the MTA with PM2.

If inertial or balloon data are not available, the GPS altitude (PM3) can still provide a reliable estimation of air data throughout the trajectory, as shown in Fig. 9. Here, the 1976 Standard Atmosphere was used throughout the trajectory. No attempt was made to extend the standard atmosphere above approximately $280,000 \mathrm{ft}(86 \mathrm{~km})$, so errors are expected at altitudes above 280,000 ft. The freestream pressure shows an increasing delta as altitude decreases. A change to the algorithm that switches from a standard atmosphere to the local altimeter setting below $18,000 \mathrm{ft}$ should improve the low altitude results. This techniques is used by all aircraft when transitioning from the high altitude environment. The deltas in the dynamic pressure are similar to the deltas provided by PM2, since dynamic pressure is provided primarily from the FADS. 


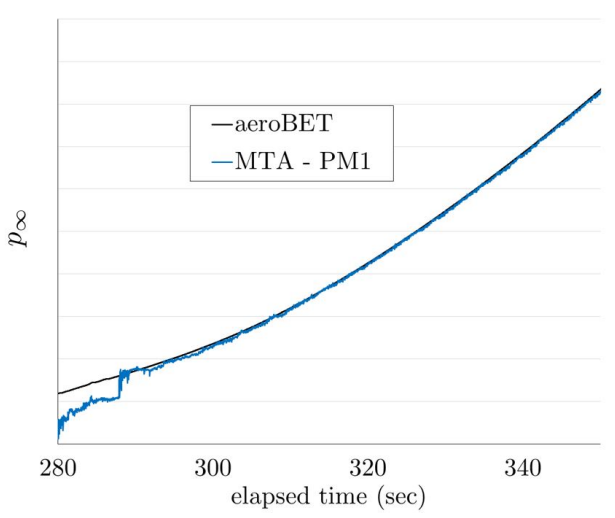

(a) Trending information

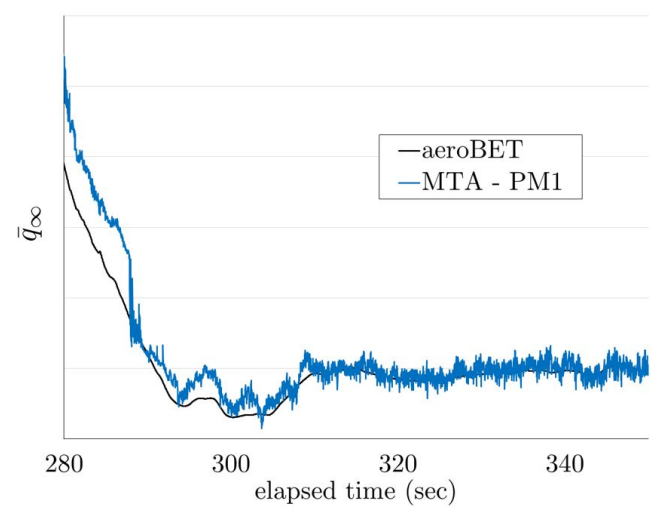

(c) Trending information

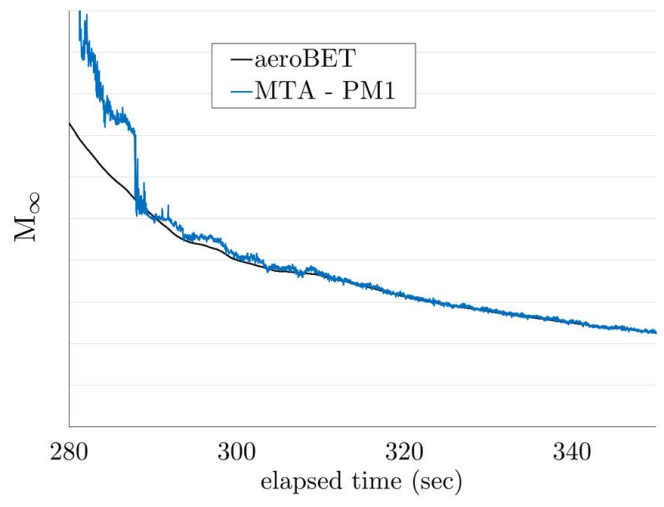

(e) Trending information

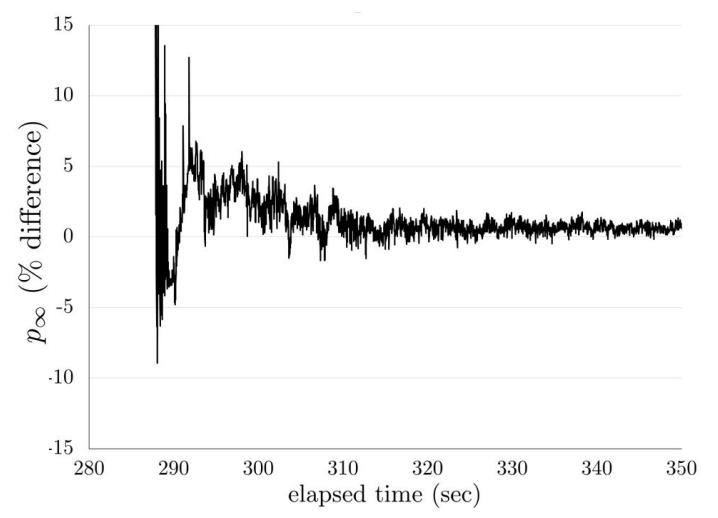

(b) Percent difference

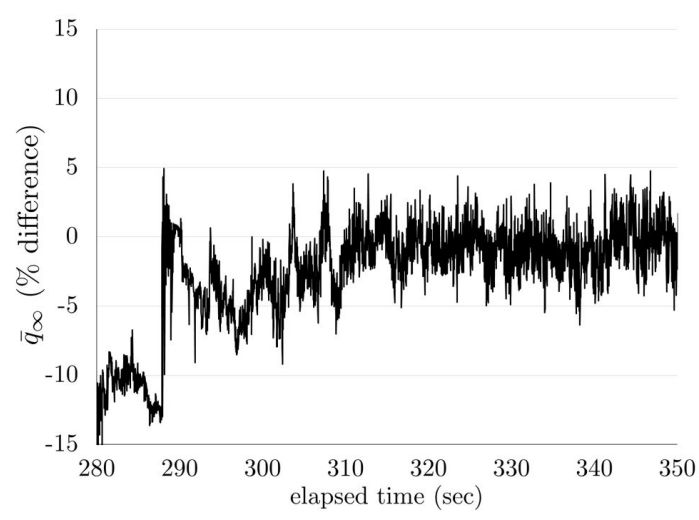

(d) Percent difference

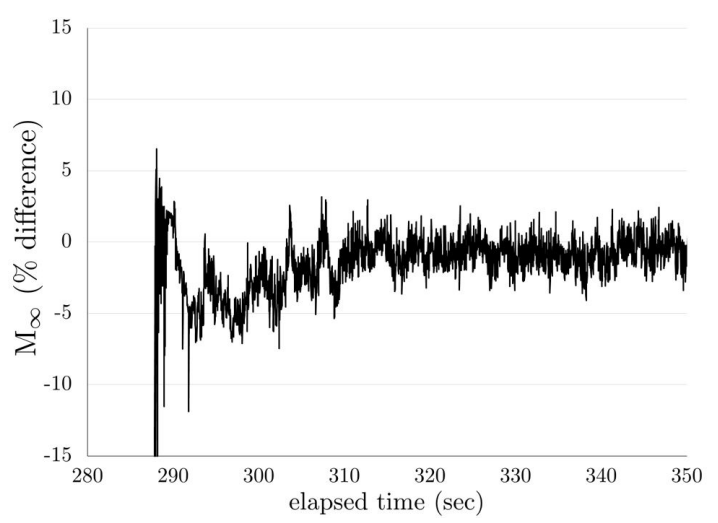

(f) Percent difference

Figure 7. Comparison of $p_{\infty}, \bar{q}_{\infty}$, and $\mathbf{M}_{\infty}$ between the aeroBET and the MTA with with Pressure Model 1 (PM1)

The Mach number is in excellent agreement once the stipulation on maximum altitude is achieved.

Since the Mach numbers are in excellent agreement with the aeroBET for both PM2 and PM3, either pressure model could be used to make the final corrections to $\alpha$ and $\beta$. Figure 10 shows the values of $\alpha$ and $\beta$ with calibrations made from PM2. For $\beta$ the trends between the MTA with PM2 and the aeroBET are nearly exact with excellent agreement throughout most of the trajectory. As seen in the pressure models, though, the predictions for $\alpha$ show a near constant delta throughout the high speed regime, while showing excellent agreement in the low speed regime. The inertial data in the aeroBET may be providing a correction, or a bias, from the pure pressure data of the MTA. While there is a delta in $\alpha$ in the high speed regime, the trends between the two models are still remarkably similar. 


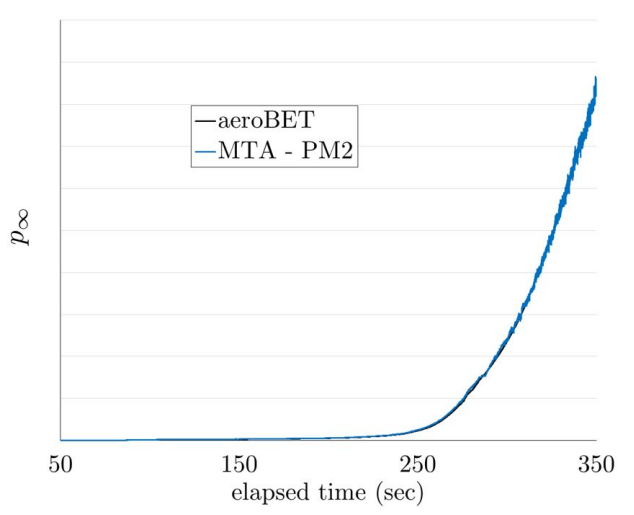

(a) Trending information

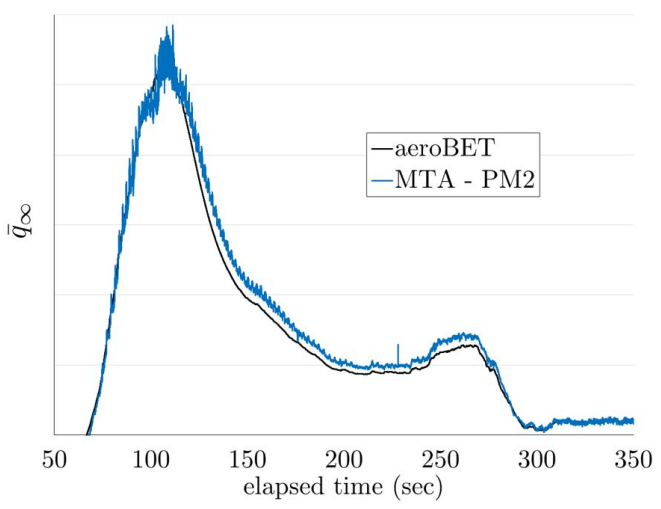

(c) Trending information

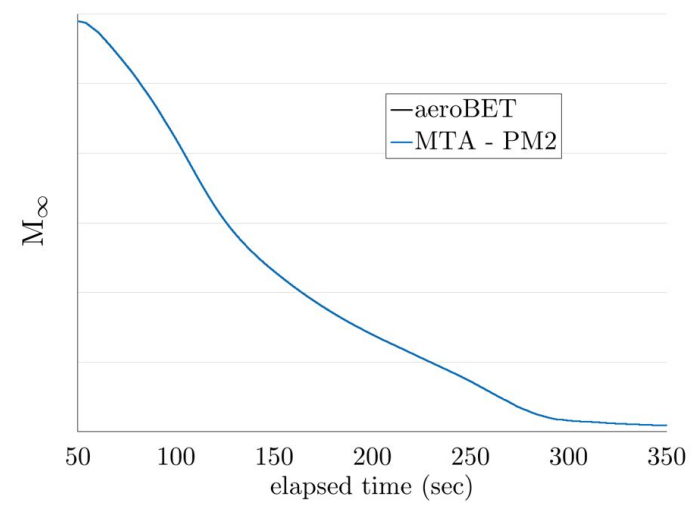

(e) Trending information

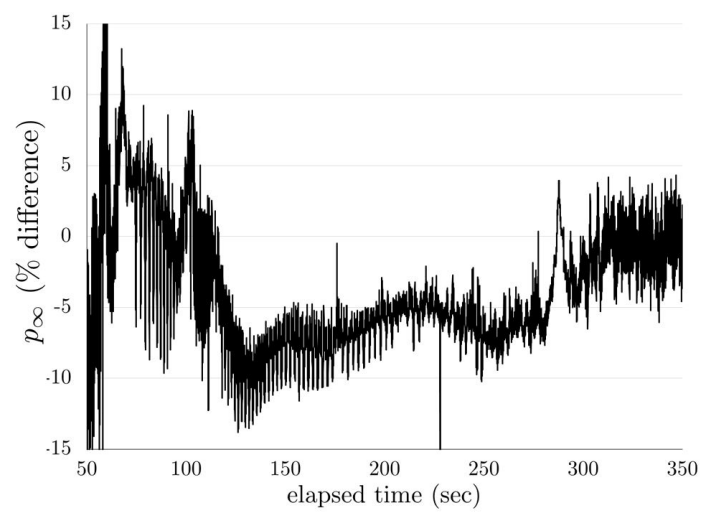

(b) Percent difference

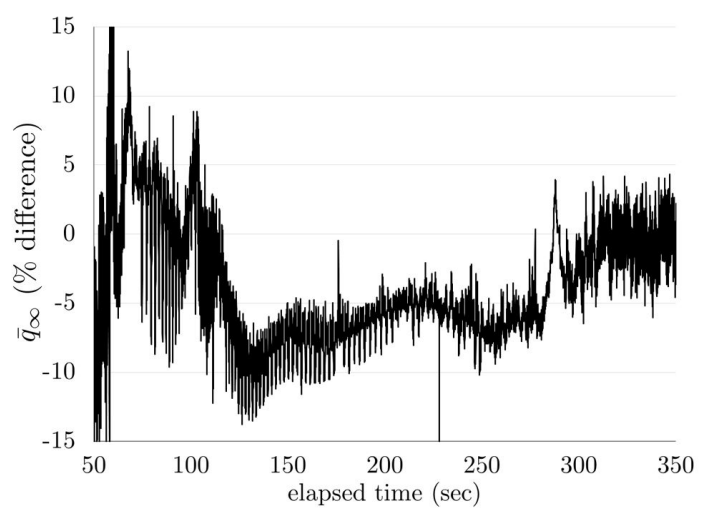

(d) Percent difference

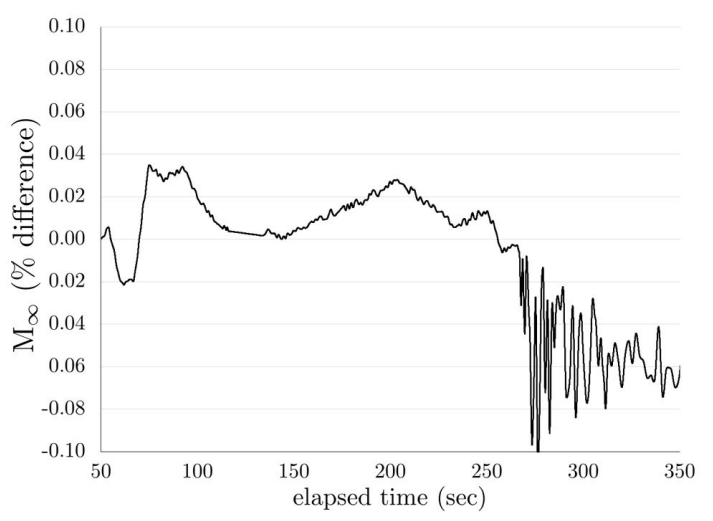

(f) Percent difference

Figure 8. Comparison of $p_{\infty}, \bar{q}_{\infty}$, and $\mathbf{M}_{\infty}$ between the aeroBET and the MTA with with Pressure Model 2 (PM2)

Finally, a comment on the applicability of using the MTA with PM2 or PM3 in a real-time setting. On average, the time to compute the $\alpha_{e}, \beta_{e}, \bar{q}_{\infty}, p_{\infty}, \mathrm{M}_{\infty}, \alpha_{t}$, and $\beta_{t}$ was on the order of $25 \%$ of the sampling time of the Orion FADS.

\section{Conclusion}

The two most prevalent algorithms for computing air data from a FADS are the LSSE and the Triples algorithm. The advantages of the LSSE include that it is considered the most accurate algorithm because all the pressure measurements are used simultaneously; it is suitable for any port configuration; and it is 


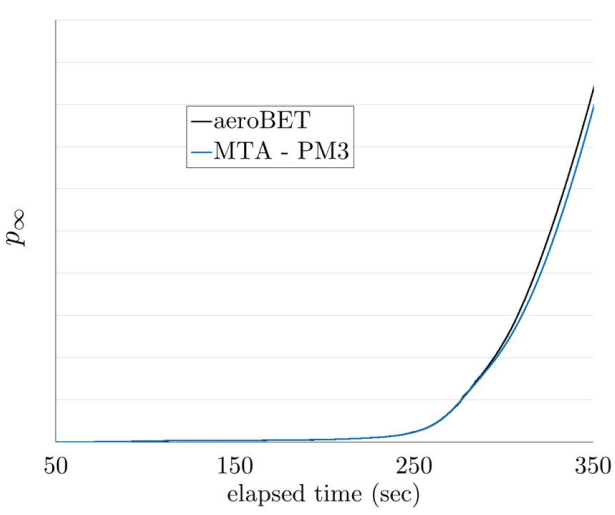

(a) Trending information

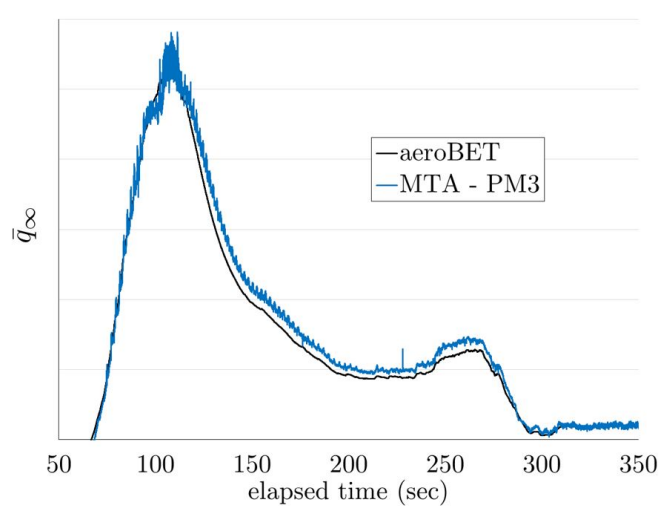

(c) Trending information

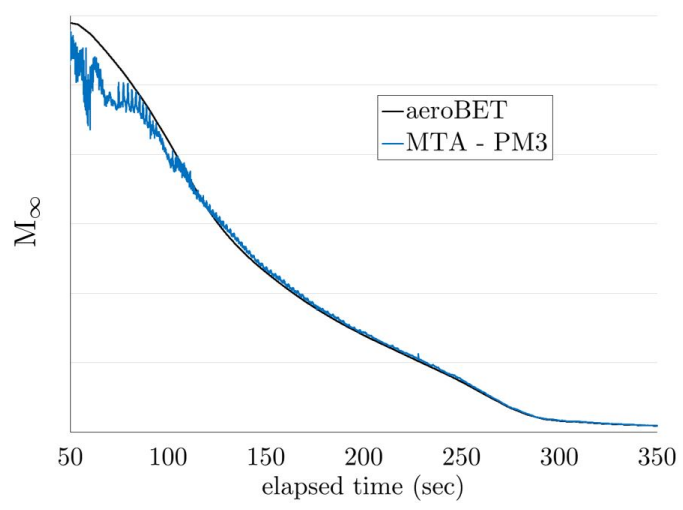

(e) Trending information

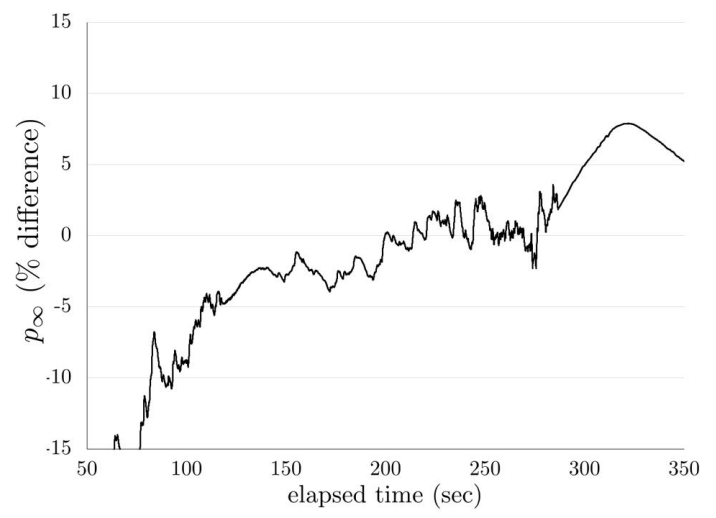

(b) Percent difference

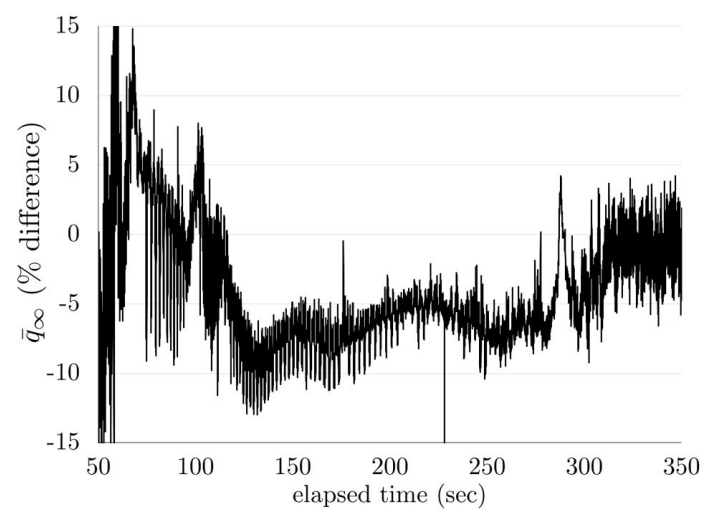

(d) Percent difference

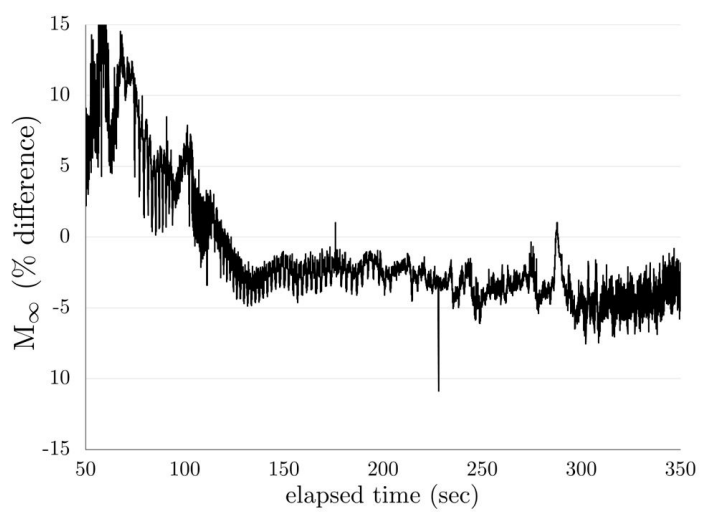

(f) Percent difference

Figure 9. Comparison of $p_{\infty}, \bar{q}_{\infty}$, and $\mathbf{M}_{\infty}$ between the aeroBET and the MTA with with Pressure Model 3 (PM3)

suitable for post-flight trajectory reconstruction. The disadvantages include complex coding and it is not always suitable for real-time calculations.

The Triples Algorithm was developed to overcome the disadvantages of the LSSE. Its advantages include none of the convergence issues experienced by LSSE; direct solution of $\alpha$ and $\beta$; simplified iterations for $p_{\infty}$ and the total pressure; and it is suitable for real-time use or post-flight trajectory reconstruction. However, the disadvantages include that it is constrained to a single port configuration, which may not be achievable due to on-board systems or structural issues; a complicated relationship between $p_{\infty}$ and the total pressure; high temperature corrections further complicate the relationship when the ratio of specific heats is no longer constant; and because the pressure model is inexact, calibration tables are required for $\alpha, \beta$, and $\varepsilon$. 


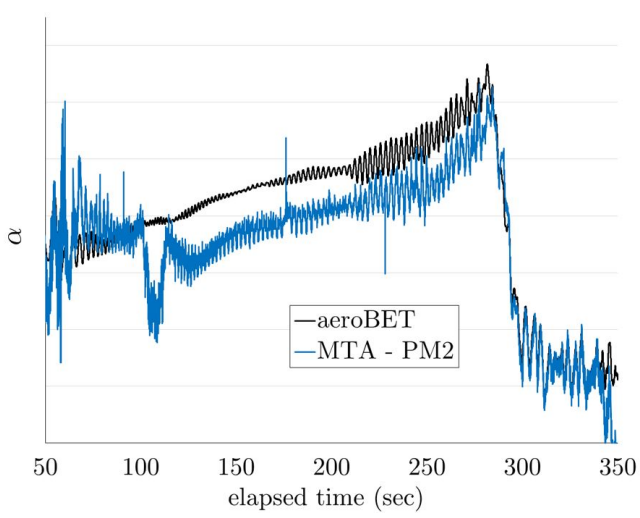

(a) Trending information

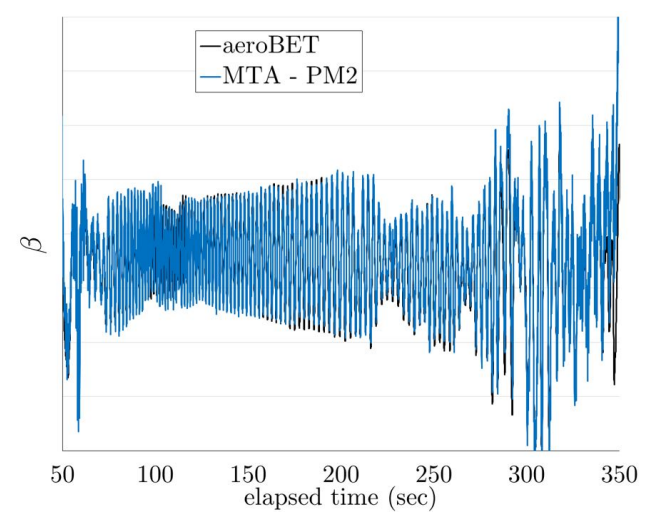

(c) Trending information

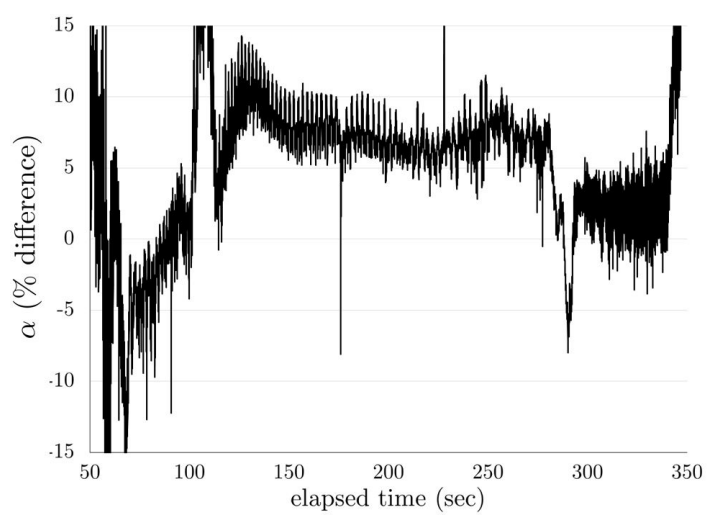

(b) Percent difference

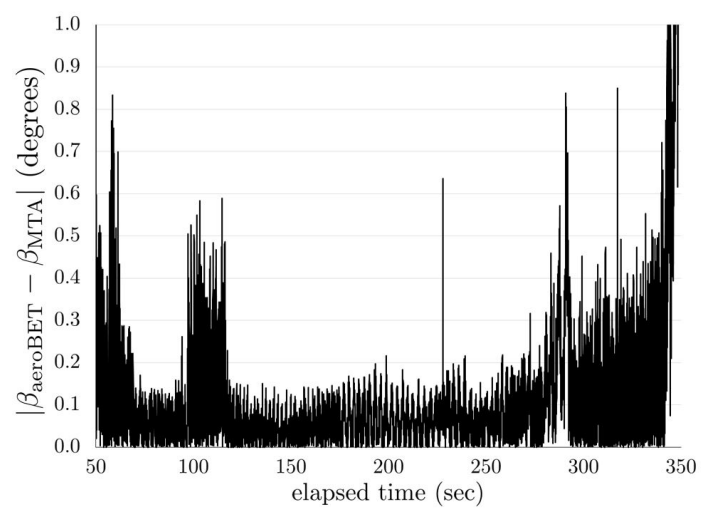

(d) Absolute difference

Figure 10. Comparison of $\alpha$ and $\beta$ between the aeroBET and the MTA with Pressure Model 2 (PM2) calibrations.

The MTA was developed to over the single configuration constraint. The pressure model, based on the LSSE, was incorporated with the MTA to simplify the pressure calculations. The advantages of the MTA include none of the convergence issues experienced by LSSE; reliable, rapidly convergent solution of $\alpha$ and $\beta$; simplified iterations for $p_{\infty}$ and $\bar{q}_{\infty}$; suitable for real-time use or post-flight trajectory reconstruction; suitable for a variety of port configurations; and no calibration required for pressure calculations. The remaining disadvantage is that, since the Triples Equation is inexact, calibration tables are still required for $\alpha$ and $\beta$.

The Orion EFT-1 flight test data validates the MTA approach for a challenging re-entry trajectory. Because of the flexibility in port arrangement, the MTA is also applicable for various aircraft and missile configurations.

\section{Acknowledgments}

After the author developed and verified the MTA, the Applied Aeroscience and CFD Branch at NASA Johnson Space Center placed the author on contract to validate the MTA against Orion EFT-1 flight test data. The validation work was performed under Booz Allen's Strategic Assessment Contract, NNJ13RB03Z. The author is especially appreciative of the numerous insights provided by members of the Applied Aeroscience and CFD Branch, particularly Ben Kirk, Ray Gomez, and Alan Schwing.

\section{Appendix}

This derivation is included in the US Patent application filed for an air data system by Booz Allen Hamilton. 
The triples equation is expanded to clearly see all the terms in $\cos ^{2} \theta_{k}$,

$$
P_{j i}\left(\cos \alpha \cos \beta \cos \lambda_{k}+\sin \beta \sin \phi_{k} \sin \lambda_{k}+\sin \alpha \cos \beta \cos \phi_{k} \sin \lambda_{k}\right)^{2}+\cdots=0,
$$

with like terms produced from $\cos ^{2} \theta_{j}$ and $\cos ^{2} \theta_{i}$. For a given value of $\beta$, define the following parameters

$$
\begin{array}{lll}
a_{i}=\cos \beta \cos \lambda_{i} & a_{j}=\cos \beta \cos \lambda_{j} & a_{k}=\cos \beta \cos \lambda_{k}, \\
b_{i}=\sin \beta \sin \phi_{i} \sin \lambda_{i} & b_{j}=\sin \beta \sin \phi_{j} \sin \lambda_{j} & b_{k}=\sin \beta \sin \phi_{k} \sin \lambda_{k}, \\
c_{i}=\cos \beta \cos \phi_{i} \sin \lambda_{i} & c_{j}=\cos \beta \cos \phi_{j} \sin \lambda_{j} & c_{k}=\cos \beta \cos \phi_{k} \sin \lambda_{k} .
\end{array}
$$

Equation (51) becomes

$$
P_{j i}\left(a_{k} \cos \alpha+b_{k}+c_{k} \sin \alpha\right)^{2}+\cdots=0
$$

which upon expanding yields

$$
P_{j i}\left(a_{k}^{2} \cos ^{2} \alpha+2 a_{k} b_{k} \cos \alpha+2 a_{k} c_{k} \cos \alpha \sin \alpha+b_{k}^{2}+2 b_{k} c_{k} \sin \alpha+c_{k}^{2} \sin ^{2} \alpha\right)+\cdots=0 .
$$

Dividing across the entire equation by $\cos ^{2} \alpha$ yields

$$
P_{j i}\left(a_{k}^{2}+2 a_{k} b_{k} \sec \alpha+2 a_{k} c_{k} \tan \alpha+b_{k}^{2} \sec ^{2} \alpha+2 b_{k} c_{k} \tan \alpha \sec \alpha+c_{k}^{2} \tan ^{2} \alpha\right)+\cdots=0 .
$$

Via the trigonometric identity $\sec ^{2} \alpha=\tan ^{2} \alpha+1$, some of the terms in Eq. (55) can be combined to obtain

$$
P_{j i}\left[\left(b_{k}^{2}+c_{k}^{2}\right) \tan ^{2} \alpha+2 a_{k} c_{k} \tan \alpha+2 b_{k} c_{k} \tan \alpha \sec \alpha+2 a_{k} b_{k} \sec \alpha+\left(a_{k}^{2}+b_{k}^{2}\right)\right]+\cdots=0 .
$$

Now invoke the following two trigonometric identities

$$
\begin{aligned}
\tan \alpha & =\frac{2 \tan \frac{1}{2} \alpha}{1-\tan ^{2} \frac{1}{2} \alpha}, \\
\sec \alpha & =\frac{1+\tan ^{2} \frac{1}{2} \alpha}{1-\tan ^{2} \frac{1}{2} \alpha},
\end{aligned}
$$

and substitute into Eq. (56) to obtain

$$
\begin{array}{r}
P_{j i}\left[\left(b_{k}^{2}+c_{k}^{2}\right) \frac{4 \tan ^{2} \frac{1}{2} \alpha}{\left(1-\tan ^{2} \frac{1}{2} \alpha\right)^{2}}+2 a_{k} c_{k} \frac{2 \tan \frac{1}{2} \alpha}{1-\tan ^{2} \frac{1}{2} \alpha}+2 b_{k} c_{k} \frac{2 \tan \frac{1}{2} \alpha\left(1+\tan ^{2} \frac{1}{2} \alpha\right)}{\left(1-\tan ^{2} \frac{1}{2} \alpha\right)^{2}}\right. \\
\left.+2 a_{k} b_{k} \frac{1+\tan ^{2} \frac{1}{2} \alpha}{1-\tan ^{2} \frac{1}{2} \alpha}+\left(a_{k}^{2}+b_{k}^{2}\right)\right]+\cdots=0 .
\end{array}
$$

Multiplying across the entire equation by $\left(1-\tan ^{2} \frac{1}{2} \alpha\right)^{2}$ yields

$$
\begin{aligned}
P_{j i}\left[4\left(b_{k}^{2}+c_{k}^{2}\right) \tan ^{2}\right. & \frac{1}{2} \alpha+4 a_{k} c_{k} \tan \frac{1}{2} \alpha\left(1-\tan ^{2} \frac{1}{2} \alpha\right)+4 b_{k} c_{k} \tan \frac{1}{2} \alpha\left(1+\tan ^{2} \frac{1}{2} \alpha\right) \\
& \left.+2 a_{k} b_{k}\left(1+\tan ^{2} \frac{1}{2} \alpha\right)\left(1-\tan ^{2} \frac{1}{2} \alpha\right)+\left(a_{k}^{2}+b_{k}^{2}\right)\left(1-\tan ^{2} \frac{1}{2} \alpha\right)^{2}\right]+\cdots=0 .
\end{aligned}
$$

Expanding the terms results in

$$
\begin{aligned}
P_{j i}\left[4\left(b_{k}^{2}+c_{k}^{2}\right) \tan ^{2} \frac{1}{2} \alpha\right. & +4 a_{k} c_{k}\left(\tan \frac{1}{2} \alpha-\tan ^{3} \frac{1}{2} \alpha\right)+4 b_{k} c_{k}\left(\tan \frac{1}{2} \alpha+\tan ^{3} \frac{1}{2} \alpha\right) \\
& \left.+2 a_{k} b_{k}\left(1-\tan ^{4} \frac{1}{2} \alpha\right)+\left(a_{k}^{2}+b_{k}^{2}\right)\left(1-2 \tan ^{2} \frac{1}{2} \alpha+\tan ^{4} \frac{1}{2} \alpha\right)\right]+\cdots=0 .
\end{aligned}
$$

Combining like terms yields

$$
\begin{aligned}
P_{j i}\left[\left(a_{k}-b_{k}\right)^{2} \tan ^{4} \frac{1}{2} \alpha+4 c_{k}\left(b_{k}-a_{k}\right) \tan ^{3} \frac{1}{2} \alpha+2\left(b_{k}^{2}+2 c_{k}^{2}+a_{k}^{2}\right) \tan ^{2} \frac{1}{2} \alpha\right. \\
\left.+4 c_{k}\left(a_{k}+b_{k}\right) \tan \frac{1}{2} \alpha+\left(a_{k}+b_{k}\right)^{2}\right]+\cdots=0 .
\end{aligned}
$$


Now define the following parameters

$$
\begin{array}{lll}
A_{i}=\left(a_{i}-b_{i}\right)^{2} & A_{j}=\left(a_{j}-b_{j}\right)^{2} & A_{k}=\left(a_{k}-b_{k}\right)^{2}, \\
B_{i}=4 c_{i}\left(b_{i}-a_{i}\right) & B_{j}=4 c_{j}\left(b_{j}-a_{j}\right) & B_{k}=4 c_{k}\left(b_{k}-a_{k}\right), \\
C_{i}=2\left(b_{i}^{2}+2 c_{i}^{2}-a_{i}^{2}\right) & C_{j}=2\left(b_{j}^{2}+2 c_{j}^{2}-a_{j}^{2}\right) & C_{k}=2\left(b_{k}^{2}+2 c_{k}^{2}-a_{k}^{2}\right), \\
D_{i}=4 c_{i}\left(b_{i}+a_{i}\right) & D_{j}=4 c_{j}\left(b_{j}+a_{j}\right) & D_{k}=4 c_{k}\left(b_{k}+a_{k}\right), \\
E_{i}=\left(a_{i}+b_{i}\right)^{2} & E_{j}=\left(a_{j}+b_{j}\right)^{2} & E_{k}=\left(a_{k}+b_{k}\right)^{2},
\end{array}
$$

which allows Eq. (61) to be written as

$$
\begin{aligned}
& P_{j i}\left(A_{k} \tan ^{4} \frac{1}{2} \alpha+\right.\left.B_{k} \tan ^{3} \frac{1}{2} \alpha+C_{k} \tan ^{2} \frac{1}{2} \alpha+D_{k} \tan \frac{1}{2} \alpha+E_{k}\right) \\
&+P_{i k}\left(A_{j} \tan ^{4} \frac{1}{2} \alpha+B_{j} \tan ^{3} \frac{1}{2} \alpha+C_{j} \tan ^{2} \frac{1}{2} \alpha+D_{j} \tan \frac{1}{2} \alpha+E_{j}\right) \\
& \quad+P_{k j}\left(A_{i} \tan ^{4} \frac{1}{2} \alpha+B_{i} \tan ^{3} \frac{1}{2} \alpha+C_{i} \tan ^{2} \frac{1}{2} \alpha+D_{i} \tan \frac{1}{2} \alpha+E_{i}\right)=0 .
\end{aligned}
$$

Finally, define

$$
\begin{aligned}
& c_{4}=P_{j i} A_{k}+P_{i k} A_{j}+P_{k j} A_{i}, \\
& c_{3}=P_{j i} B_{k}+P_{i k} B_{j}+P_{k j} B_{i}, \\
& c_{2}=P_{j i} C_{k}+P_{i k} C_{j}+P_{k j} C_{i}, \\
& c_{1}=P_{j i} D_{k}+P_{i k} D_{j}+P_{k j} D_{i}, \\
& c_{0}=P_{j i} E_{k}+P_{i k} E_{j}+P_{k j} E_{i},
\end{aligned}
$$

and the triples equation can now be written as

$$
c_{4} \tan ^{4} \frac{1}{2} \alpha+c_{3} \tan ^{3} \frac{1}{2} \alpha+c_{2} \tan ^{2} \frac{1}{2} \alpha+c_{1} \tan \frac{1}{2} \alpha+c_{0}=0 .
$$

\section{References}

\footnotetext{
${ }^{1}$ Karlgaard, C. D., Beck, R. E., O‘Keefe, S. A., Siemers, P. M., White, B. A., Engelund, W. C., and Munk, M. M., "Mars entry atmospheric data system modeling and algorithm development," AIAA 2009-3916, San Antonio, TX, June 2009.

${ }^{2}$ Bibb, K. L., Cassidy, L. D., and Schwing, A. M., "Orion EFT-1 FADS-Based Trajectory Reconstruction," AIAA 20163254, Washington, D.C., June 2016.

${ }^{3}$ Wolf, H., Henry, M.W., and Siemers, Paul M., III, "Shuttle Entry Air Data System (SEADS): Optimization of Preflight Algorithms based on Flight Results," AIAA 88-2053, May 1988.

${ }^{4}$ Weiss, S., "Comparing three algorithms for modeling flush air data systems," AIAA-2002-13830, Reno, NV, Jan. 2002.

${ }^{5}$ Whitmore, S. A., Cobleigh, B. R., and Haering, Jr., E. A., "Stable Algorithm for Estimating Airdata from Flush Surface Pressure Measurement," June 2001.

${ }^{6}$ Colbeigh, B. R., Whitmore, S. A., and Haering, Jr., E. A., "Flush Airdata Sensing (FADS) System Calibration Procedures and Results for Blunt Forebodies," NASA/TP-1999-209012, Nov. 1999.

${ }^{7}$ Whitmore, S. A., Moes, T. R., and Larson, T. J., "Preliminary Results from a Subsonic High Angle-of-Attack Flush Airdata Sensing (HI-FADS) System: Design, Calibration, and Flight Test Evaluation," NASA TM 101713, Jan. 1990.

${ }^{8}$ Dutta, S. and Braun, R. D., "Cram $\backslash$ ' $\{$ e $\}$ rRao Lower-Bound Optimization of Flush Atmospheric Data System Sensor Placement," Journal of Spacecraft and Rockets, Vol. 51, No. 6, Nov. 2014, pp. 1773-1788.

${ }^{9}$ Zipfel, P. H., Modeling and Simulation of Aerospace Vehicle Dynamics, AIAA Education Series, American Institute of Aeronautics and Astronautics, Reston, VA, 2000.

${ }^{10}$ Whitmore, S. A. and Ellsworth, J. C., "Simulation of a Flush Air-Data System for Transatmospheric Vehicles," Journal of Spacecraft and Rockets, Vol. 45, No. 4, July 2008, pp. 716-732.

${ }^{11}$ Whitmore, S. A. and Moes, T. R., "Failure Detection and Fault Management Techniques for Pneumatic High-Angleof_Attack Flush Airdata Sensing (HI-FADS) System," NASA TM 4335, Jan. 1992.

${ }^{12}$ Karlgaard, C., Kutty, P., Shidner, J., Schoenenberger, M., and Munk, M., "Mars Entry Atmospheric Data System Trajectory Reconstruction Algorithms and Flight Results," American Institute of Aeronautics and Astronautics, Jan. 2013.
} 